\title{
Paper
}

\section{Adaptive Load Balancing Ad Hoc Routing Scheme Inspired by True Slime Mold}

\author{
Hiroshi Katada, Taku Yamazaki, and Takumi Miyoshi \\ Shibaura Institute of Technology, Saitama, Japan
}

https://doi.org/10.26636/jtit.2019.129518

\begin{abstract}
Engineering neo-biomimetics, i.e. imitation models based on body structures and behavior of living organisms, relied upon to solve complex problems, have been studied in various fields. In distributed networks, such as ad-hoc networks and wireless sensor networks, the behavior of a variety of true slime molds which are capable of constructing multipath flow networks based on the amount of body, has been studied. Ad hoc networks only consist of mobile terminals (nodes) that can relay packets along an established route. However, link relations and the available bandwidth of the nodes change dynamically due to the mobility of nodes. In addition, the speed of communication between nodes also varies due to node positions and their communication-related quality. Thus, practical use of ad-hoc networks still remains an issue, because it is difficult to establish stable routes under such environments. This study aims to propose an adaptive load balancing routing technique that adaptively diversifies the transmission paths based on the available bandwidth, residual battery life, and the data transmission volume, by applying a mathematical model of slime mold routing, known as the physarum solver. We confirm the effectiveness of its adaptive behavior in dynamic environments using computer simulations.
\end{abstract}

Keywords-adaptive ad hoc routing, ad hoc network, engineering neo biomimetic, physarum solver, true slime mold.

\section{Introduction}

Life forms change and optimize their structures and behavioral patterns in the course of evolution. Recently, biomimetic technologies used to design artifacts inspired by specific abilities and structures have been studied [1]. Primary examples of the application of biomimetic technologies include swimsuits inspired by the skin structure of sharks [2], and nylon fiber inspired by the fiber structure of cotton [3]. In the field of network research, various bioinspired mechanisms, such as multiple route optimization inspired by the feeding behavior of physarum [4], shortestpath route optimization inspired by the feeding behavior of ants [5], and a synchronization mechanism inspired by the synchronous behavior of fireflies, have also been studied [6]. The features of Physarum Polycephalum, which is a variety of physarum, are applied to design a routing protocol. Please note that Physarum Polycephalum is simply referred to as physarum below. As far as the nature of physarum's feeding process is concerned, it has been confirmed that physarum creates a tube for nutritional transport, utilizes its own body and connects foods using the tube when it finds multiple baits at different places. It also has been confirmed that the number of constructed paths varies depending on the amount of liquid that is the constituent of physarum. Additionally, physarum has a negative phototaxis, which means that physarum can limit the area over which to spread its body [7]. Therefore, physarum may optimize trade-offs between efficiency and stability of nutritional transport paths, which means that is characteristics may be applied to the selection of a relay node in wireless multi-hop networks. Those characteristics may be applied, for instance, in an ad hoc network that consists of mobile wireless terminals (nodes) only, without relying on a base station [8]. The ad hoc network finds exceptional use in an area where a base station cannot be placed or has been destroyed due to a disaster. In ad hoc networks, nodes can communicate with each other by relaying packets. Such multi-hop communication may be performed when relay nodes exist, even if they are located outside the communication range. However, due to such factors as node mobility, low battery states, radio interference, dynamic topology changes, and network stability, degradations may occur. One solution includes the use of a multipath routing protocol that can alleviate the impact of the dynamic changes by simultaneously using multiple paths.

In addition, in recent wireless communication media, such as IEEE 802.11 [9], [10], offer a functionality that may select the appropriate transmission speed between nodes [11]. Some research concerning the rate adaptation algorithm has been conducted as well [12]-[14]. Especially, multiple input and multiple output (MIMO) systems [15] which drastically improve the transmission speed have been proposed by using multiple transmit and receive antennas. As a result, the speed of communication between nodes varies widely due to the variation in communication quality caused by node mobility, radio interference and other factors experienced in real life environments. Hence, the effect of varying communication speed is approached adaptively as well. 
In this study, we propose a multipath ad hoc routing method by applying a mathematical model inspired by the path finding ability typical of physarum, known as the physarum solver (PS) [16]. By applying PS, the proposed method constructs multiple paths and adaptively allocates bandwidth to such paths based on the data transmission volume, bandwidth available within each link and the residual battery level of each node.

\section{Related Work}

Physarum consists of a stretchable tube and viscous liquid flowing through it. The tube is a path connecting multiple baits, and becomes thinner or thicker in response to flow rate fluctuations. In addition, the tube has an upper limit to its thickness. Figure 1 shows an example of a route constructed by physarum in a maze. First, physarum spreads its tube over the entire maze. Next, it selects paths where food has been located. Then, the tube gradually becomes thinner as the liquid flow rate decreases, when the path becomes relatively longer than the remaining paths. In addition, since no flow takes place in a blind tube, the tube degenerates relatively quickly. Therefore, physarum prioritizes shorter and continuous paths, and the number of remaining paths varies based on the total amount of liquid constituting physarum. In addition to the above properties, physarum moves away from sources of light (negative phototaxis), and therefore the tube becomes thinner along the segment exposed to light. If the path is disrupted by an external factor, physarum takes a detour path to reallocate the flow of the disrupted path to other paths to avoid path disruption of connectivity between the baits. Therefore, physarum may adjust the efficiency and stability of nutritional transport paths.

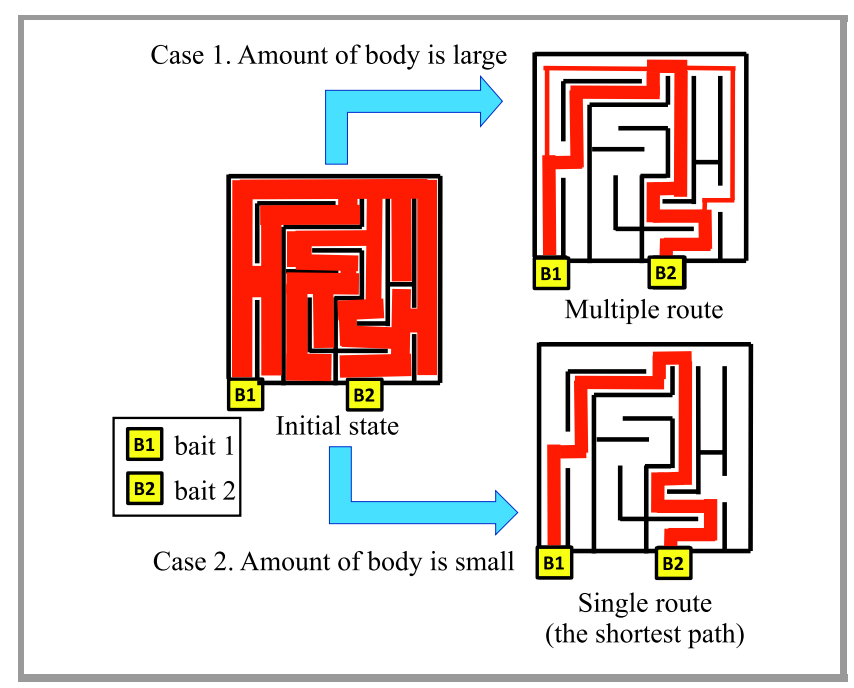

Fig. 1. Behavior of slime mold in the maze.

An experiment [17], in which the behavior of physarum on a railway network was observed, has been conducted. In the experiment, an agar medium imitating the railway network in the Kanto region was prepared, and bait was placed at positions that correspond to major cities in the region. Then, physarum was placed in the position that representing Tokyo, and the behavior of physarum was observed. In the experiment, the light quantity is adjusted according to the elevation and position of the river to imitate the topography of the Kanto region. In the experimental results, physarum spreads in approximately half a day, and it becomes clear that the paths similar to the current railway network are constructed between baits. This result has revealed that the efficiency and stability of transport paths designed by humans and physarum are similar.

\subsection{Physarum Solver}

Physarum solver (PS) [16] is a mathematical model which focuses on the feeding behavior of physarum when physarum constructs a route between baits. PS calculates the flow rate of each tube based on the total amount of liquid in physarum, as well as on the length, thickness and pressure loss of each tube. In the initial state, PS calculates the pressure loss in each tube based on the total amount of liquid, length and thickness of the tube. Thereafter, the flow rate of each tube is temporarily determined in accordance with the pressure loss of the tube, and then the flow rate of the tube changes as the thickness varies. By iterating such a process, the flow rate and thickness of the tube converge to an appropriate value, and finally the tubes that remain are based on the total amount of liquid. Furthermore, by setting the parameters of phototaxis, it is possible to control the thickness of a tube independently of the flow rate.

The functioning principle of PS, with baits placed at two locations, is shown below. Here, PS deals with tubes that have branch points $i$ and $j$ at both ends.

1. PS calculates the pressure loss of the tube between $i$ and $j$ based on the flow rate of the tube, as calculated by Eq. (1), and based on the flow conservation law of the tube, calculated by Eq. (2):

$$
\begin{gathered}
Q_{i j}(t)=\frac{D_{i j}(t)}{L_{i j}}\left[p_{i}(t)-p_{j}(t)\right], \\
\sum_{i} Q_{i j}=\left\{\begin{array}{cc}
-Q_{\text {all }}, & j=\text { bait } 1 \\
Q_{\text {all }}, & j=\text { bait } 2 \\
0, & \text { otherwise }
\end{array}\right.
\end{gathered}
$$

where $Q_{\text {all }}$ is the total amount of liquid; $D_{i j}(t)$ and $L_{i j}$ are the thickness and length of the tube, respectively; $p_{i}(t)-p_{j}(t)$ is the pressure loss of the tube.

2. By substituting the derived pressure loss into Eq. (1), the flow rate of each tube $Q_{i j}(t)$ is temporarily determined, and thereby a shorter and thicker tube allows a larger flow rate. However, due to pressure loss in the blind tube, the flow rate also becomes low.

3. By substituting the flow rate of each tube in Eq. (3), PS updates the tube thickness:

$$
D_{i j}(t+\delta t)=D_{i j}(t)+\delta t\left\{f\left(\left|Q_{i j}(t)\right|\right)-a D_{i j}(t)\right\},
$$


Here,

$$
f\left(\left|Q_{i j}(t)\right|\right)=\frac{\left|Q_{i j}(t)\right|^{\mu}}{1+\left|Q_{i j}(t)\right|^{\mu}}, \quad \mu>1 .
$$

$a$ is a parameter expressing the extent of phototaxis and it controls the degeneration speed of the tube. When the flow rate is high or low, variations in the thickness of the tube decrease since it changes based on a sigmoid curve. $\mu$ is a gradient of the sigmoid function. It is the convergence speed of the thickness of the tube.

4. PS re-enters the updated tube thickness into Eq. (1).

By iterating the above mentioned procedure, PS converges the route between baits.

As described above, PS operates by treating the maze as a flow network. Therefore, it is possible to use PS as a routing solution in computer and transportation networks.

Car navigation has been proposed as one application of PS [18]. In this method, the system determines a route along an interstate highway between Seattle and Houston, USA, based on PS. PS derives a single route with the shortest mileage, when no trouble in the transportation network is encountered. When routes are congested, PS obtains the route with the shortest time of travel, by changing the value of $a$ according to the traffic volume. Moreover, if an accident takes place in the middle of the route, PS identifies the optimum detour route to avoid the section of the road where the accident has taken place.

\subsection{Physarum-based Routing Scheme}

A Physarum-based routing scheme (P-bRS) has been proposed that applies PS to routing in wireless sensor networks (WSN) [19]. The network model of P-bRS assumes that a multi-hop WSN consists of static sensor nodes and a single mobile sink node, which are uniformly arranged in a two-dimensional space. The network model also assumes that each sensor node may obtain information about the position of all sensor nodes and their residual battery levels. The sink node broadcasts its current position periodically to the surrounding sensor nodes while moving along a specific route.

In P-bRS, each parameter of PS is redefined to apply PS to WSN. Equation (1) and Eq. (3) are changed to Eq. (4) and Eq. (6), respectively:

$$
\begin{gathered}
Q_{i j}(t)=\frac{D_{i j}(t)}{L_{i j}}\left[p_{i}(t)-p_{j}(t)\right] \\
=\frac{k \mathrm{ER}_{j}(t)+(1-k) \cos \theta_{j i d}}{L_{i j}}=\frac{P_{i j}(t)}{L_{i j}}, \\
\theta_{j i d}=\arccos \frac{L_{i j}^{2}+L_{i d}^{2}-L_{j d}^{2}}{2 L_{i j} L_{i d}} \\
P_{i j}(t+\delta t)=P_{i j}(t)+\delta t\left\{\left(Q_{i j}(t)\right)^{\mu}-P_{i j}(t)\right\}
\end{gathered}
$$

where: $Q_{i j}(t)$ is the virtual data packet size between node $i$ and $j, L_{i j}$ is the Euclidean distance between node $i$ and $j$, $\mathrm{ER}_{j}$ is the residual battery level of node $j, \theta_{j i d}$ is the angle of deviation that is derived from the cosine formula, and its range is $\left[-\frac{\pi}{2} \leq \theta_{i j d} \leq \frac{\pi}{2}\right]$. Figure 2 shows an example of next-hop selection in P-bRS. As shown in the figure, nodes closer to the sink node have a smaller angle of deflection. $D_{i j}(t)$, which is defined as the link quality, is omitted in Eq. (4) because the model assumes that $D_{i j}(t)$ is always constant. Coefficient $k$ is used to adjust the weight of the residual battery level and the angle of deviation. Therefore, P-bRS constructs a route based on both the residual battery level and the angle of deviation to replace the pressure loss in PS.

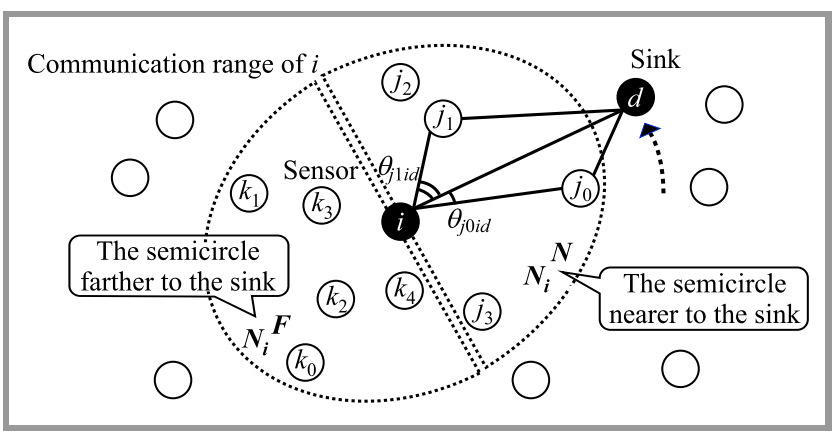

Fig. 2. Example of topology in P-bRS.

The operating principle of P-bRS in the scenario in which data is transmitted from sensor node $i$ to sink node $d$ is shown below. When a data transmission request is placed, sensor node $i$ divides its communication range into semicircle $N_{i}^{N}$, which is closer to the sink node, and semicircle $N_{i}^{F}$, which is further from the sink node, based on the position information received from the sink node. Thereafter, node $i$ calculates $P_{i j}(t)$ for node $j$ that belongs to $N_{i}^{N}$ using Eq. (4)-Eq. (6). Here, node $i$ gives order $j_{x}$, which is arranged by $P_{i j}(t)$ in a descending order. Node $i$ selects the node $j_{0}$ as a relay node and transmits a control packet to the node $j_{0}$. Node $j_{0}$ transmits an acknowledgement packet (ACK) to node $i$ after receiving the control packet. Then, if the node $j_{0}$ satisfies $N_{j_{0}}^{N}=\phi$, it does not transmit ACK. If node $i$ has not received ACK from node $j_{0}$ after a certain period, node $i$ selects the relay node $j_{x}$ of the smallest $x$ that satisfies $\left|\theta_{j_{0} i d}-\theta_{j_{x} i d}\right| \geq \frac{\pi}{2}$ and $N_{j_{x}}^{N} \neq \phi$, and transmits a control packet. If node $j_{x}$ which satisfies these conditions does not exist, node $i$ calculates $P_{i k}(t)$ for node $k$, which belongs to $N_{i}^{F}$. Here, node $i$ gives an order $k_{y}$ to the node $k$, which is arranged by $P_{i j}(t)$ in an ascending order. Node $i$ selects relay node $k_{y}$, as in case of selection of node $j_{x}$, and retransmits the control packet to $k_{y}$. Therefore, $\mathrm{P}-\mathrm{bRS}$ constructs a route to avoid nodes in low density areas, thus preventing an increase in the transmission delay. Node $i$ initiates data transmission after receiving ACK from the node $j_{x}$ or $k_{y}$ to finish its routing process. By iterating the process described above until the data reaches the sink node, P-bRS constructs a route based on the Euclidean distance to the sink node, angle of deviation, and 
residual battery level, while avoiding the low node density area.

The results of computer simulation evaluations have confirmed that P-bRS improves the efficiency of the battery use and transmission delay of the nodes compared to the previous method. However, P-bRS does not consider bandwidth use, as derived from the communication performed by other source nodes. Therefore, data collection may be difficult when congestion occurs in the area closest to the sink node in a scenario with considerable communication volume.

\section{Adaptive Load Balancing Routing Inspired by True Slime Mold}

Now we propose an adaptive load balancing routing mechanism inspired by true slime mold model for ad hoc networks, which is capable of constructing multiple paths based on data transmission volume, available bandwidth of each link and the residual battery level of each node, by applying PS to a dynamic network.

Figure 3 presents an overview of PS in the proposed method. Here, the volume of data transmitted between end-to-end nodes, data transfer rate on each link and the transmission delay on each link in the figure correspond to the total amount of liquid, the flow rate of each tube and the length of each tube in physarum, respectively. Additionally, the bandwidth utilization rate is the rate occupied by the current bandwidth used within the maximum bandwidth of a link. The bandwidth occupancy rate is the part of the bandwidth occupied by the data transmission volume requested by the source node, within the maximum bandwidth of the link. The bandwidth occupancy rate corresponds to the thickness of the tube in physarum.
Each node periodically calculates the bandwidth occupancy rate and the transmission delay time of all the links from the bandwidth utilization rate and the maximum bandwidth.

The operating principle of the proposed method is shown below.

1. When a data transmission request occurs, the source node calculates the data packet transfer time $p_{i j}(t)$ by Eqs. (7)-(8) from the transmission data size $Q_{\text {all }}$, bandwidth occupancy rate $D_{i j}(t)$, and the transmission delay $L_{i j}(t)$ between nodes $i$ and $j$ :

$$
\begin{gathered}
Q_{i j}(t)=\frac{D_{i j}(t)}{L_{i j}(t)} p_{i j}(t), \\
\sum_{i} Q_{i j}=\left\{\begin{array}{cl}
-Q_{\text {all }}, & j=\text { source } \\
Q_{\text {all }}, & j=\text { destination } \\
0, & \text { otherwise }
\end{array}\right.
\end{gathered}
$$

2. The source node calculates the transfer data size $Q_{i j}(t)$ on the link $i-j$ from the data packet transfer time by Eq. (7). Consequently, larger data transfer volumes are allocated to the link that has a larger bandwidth occupancy rate and a smaller transmission delay.

3. The bandwidth occupancy rate is updated by:

$$
D_{i j}(t+\delta t)=D_{i j}(t)+\delta t\left\{f\left(\left|Q_{i j}(t)\right|\right)-a D_{i j}(t)\right\} \text {. }
$$

Here,

$$
f\left(\left|Q_{i j}(t)\right|\right)=\frac{\left|Q_{i j}(t)\right|^{\mu}}{1+\left|Q_{i j}(t)\right|^{\mu}}, \quad \mu>1 .
$$

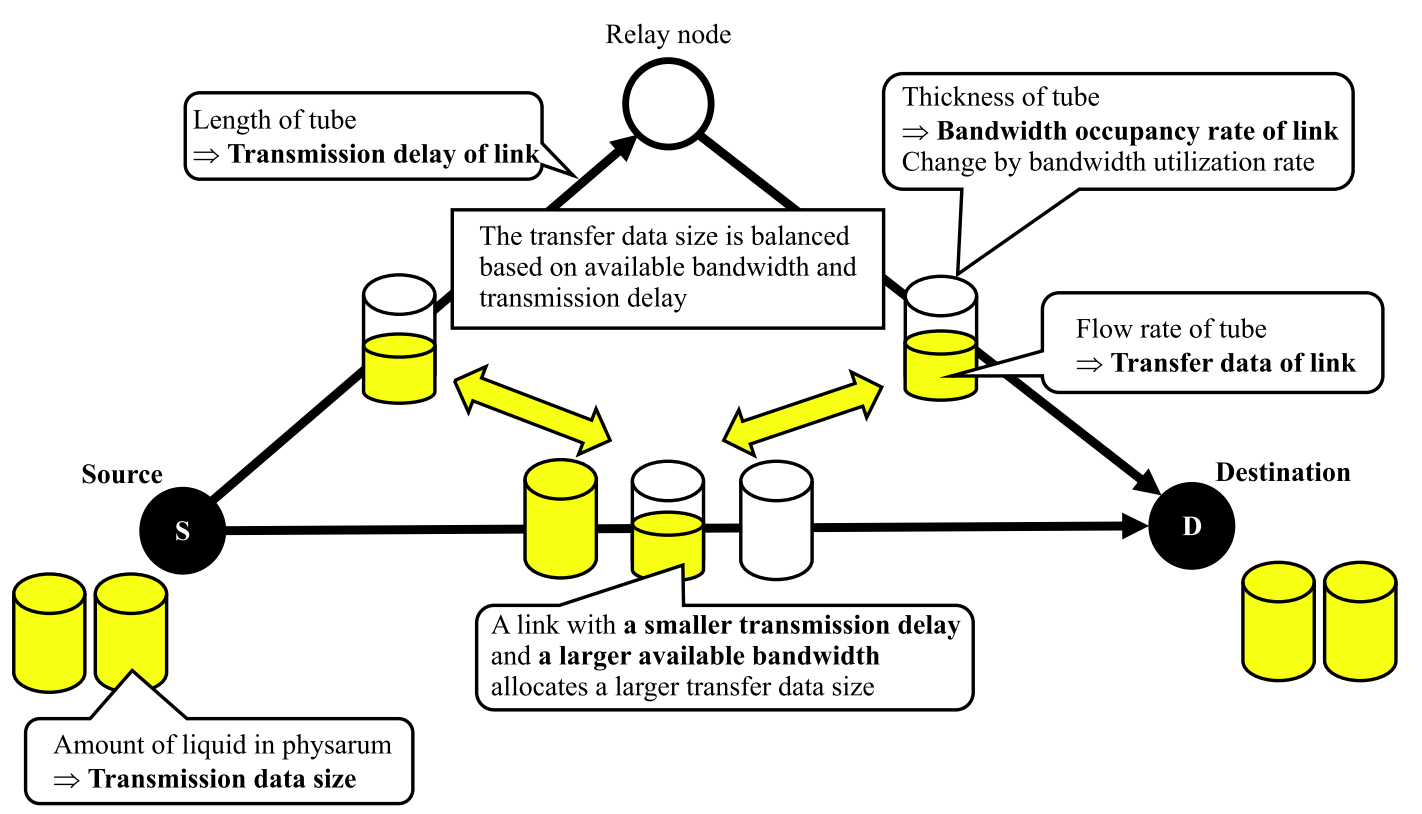

Fig. 3. Overview of PS in the proposed method. 
$\delta t\left\{f\left(\left|Q_{i j}(t)\right|\right)-a D_{i j}(t)\right\}$ in Eq. (9) represents the variation in the bandwidth occupancy rate after $\delta t$. The bandwidth occupancy rate asymptotically converges to specific values because the variation becomes small when using a sigmoid function, even if the transfer data size is large. Therefore, as the data transmission volume increases, the number of links with saturated bandwidth occupancy rates increases as well. The damping coefficient $a$ in Eq. (9) is a parameter for changing the bandwidth occupancy rate independently of the data transfer volume. It is normally set to 1 . It enables a path to be constructed that prioritizes a relay node with a large amount of residual battery level, by changing the damping coefficient according to the residual battery life of the node. Thereby, it may improve stability of the route to avoid route disruption due to the loss of battery charge.

The source node iterates the above calculation until the data transfer volume on each link converges. After convergence, the source node begins data packet transmission based on the data transfer volume of each link, while distributing the traffic among the links. Therefore, the proposed method may transmit data along a constructed route based on the data transmission volume and available bandwidth.

\section{Performance Evaluation}

\subsection{Simulation Environment}

Simulations evaluate the behavior and performance of the proposed method using software relying on the $\mathrm{C}++$ programming language. First, performed a simulation based on the topology shown in Fig. 4. In simulations 1 to 3 , the maximum bandwidth of each link was set to $11 \mathrm{Mbps}$ on the assumption that the nodes used IEEE 802.11b [9] as the wireless communication medium, and the initial bandwidth utilization rate was set to $0 \%$. Meanwhile, in simulations 4.1 and 4.2, the maximum bandwidth of each link was set to several fixed values based on the rates supported by IEEE $802.11 \mathrm{ac}$ [10] and the initial bandwidth utilization rate was

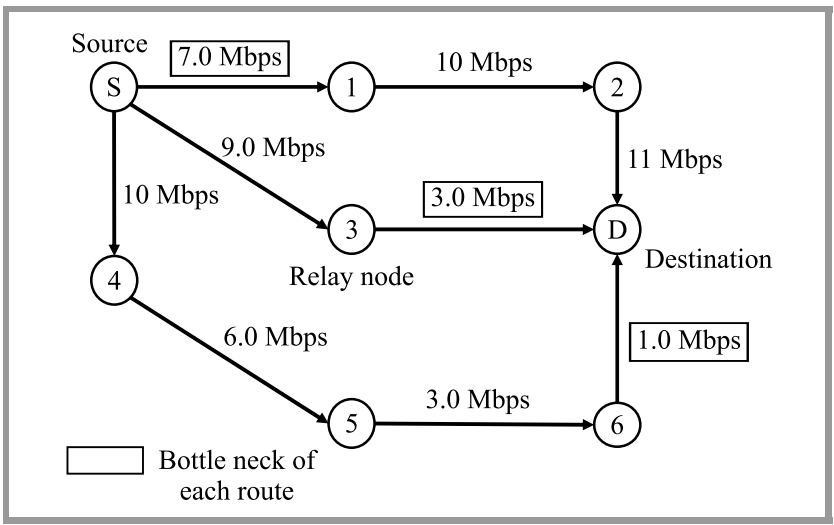

Fig. 4. Simulation topology utilizing $802.11 \mathrm{~b}$ and the available bandwidth when the iteration count is 2000 (default is $11 \mathrm{Mbps}$ ). set to $0 \%$. We observed and evaluated the transition of the allocated data transfer volume on each path, and used it as an evaluation index, under several different conditions.

In this paper, we completed the following simulations under different conditions.

In simulation 1, we evaluate the effect of varying available bandwidth using the topology as shown in Figs. 4 and 5. We assume that the available bandwidth of each link changes due to radio interference in the simulation. Therefore, the maximum bandwidth of each link and bandwidth utilization rate of each link varies within the range of 1 and $11 \mathrm{Mbps}$ and $1 \%$ to $100 \%$, respectively, when the number of iterations of calculations reaches 2000 and 4000. Figures 4 and 5 show the simulation topology and available bandwidth on each link when the number of calculation iterations reaches 2000 and 4000 .

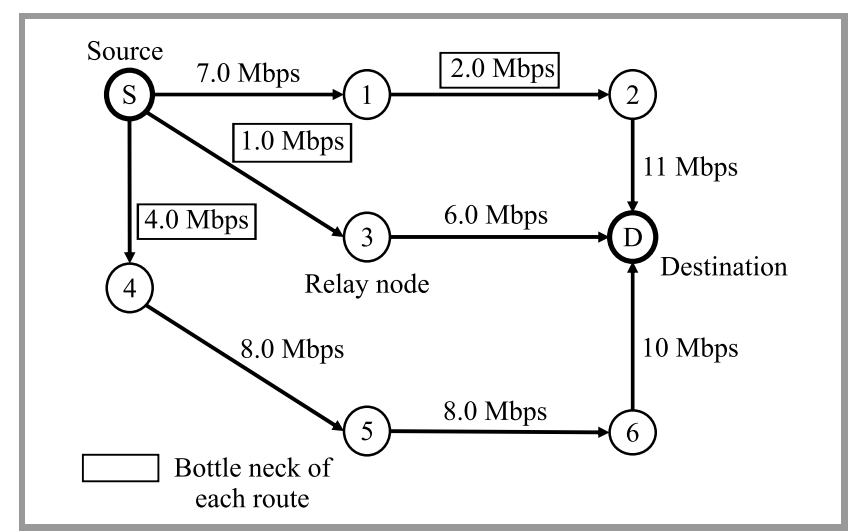

Fig. 5. Simulation topology utilizing $802.11 \mathrm{~b}$ and the available bandwidth when the iteration count is 4000 .

In simulation 2, we evaluate the effect of varying data transfer volume using the same topology of simulation 1 that is shown in Fig. 4. We assume the data transmission volume is changed before determining a route. Therefore, the data transfer volume is set to $5 \mathrm{MB}$ at the initial time. Then, when the number of calculation iterations reaches 2000 , the data transfer volume is changed to $10 \mathrm{MB}$, and when the number of iterations reached 4000 , it changed to $5 \mathrm{MB}$.

In simulation 3, we evaluate the effect of varying the residual battery level of the node using the topology as shown in Fig. 4. In the topology of Fig. 4, node 3 transmits data packets with a higher frequency in comparison with other nodes since it is located along the shortest path. Namely, the battery consumption of the node also increases in comparison with other nodes due to the reason referred to above. Assuming that node 3 exceeds its battery power available when the number of iterations reaches 2000 and 4000 , node 3 adds one to its damping coefficient. Additionally, if the bandwidth occupancy rate of the link decreases as the residual battery level of node 3 becomes lower, it is expected that the data transfer volume and the battery consumption of the nodes along the second shortest path S-1-2-D increase. Thus, node 1 adds one to its damping co- 
efficient every 1000 iterations after the number of iterations has reached 6000 .

In simulation 4.1, we evaluate the effect of varying available bandwidth widely using the topology as shown in Figs. 6 and 7. Therefore, the simulation assumes a fluctuation of bandwidth when using IEEE 802.11ac as the wireless communication medium. Here, we assume IEEE 802.11ac that adopted OFDM-MIMO as the primary modulation scheme, 64 quadrature amplitude modulation 5/6 as the secondary modulation scheme, and the guard interval is $800 \mathrm{~ns}$. The maximum number of enable streams of each node is 8 . Under the above conditions, the theoretical values of the communication speed are 292.5, 585, 877.5 , and $1170 \mathrm{Mbps}$ when the number of antennas is 1 , 2,3 , and 4, respectively. Additionally, we suppose that the bandwidth occupancy rate on each link is $100 \%$ in the simulation. Hence, we change the transmission speed stepwise based on the rate candidates when the number of calculation iterations reaches 2000 and 4000. Figures 6 and 7 show the simulation topology and available bandwidth on each link when the number of calculation iterations reaches 2000 and 4000.

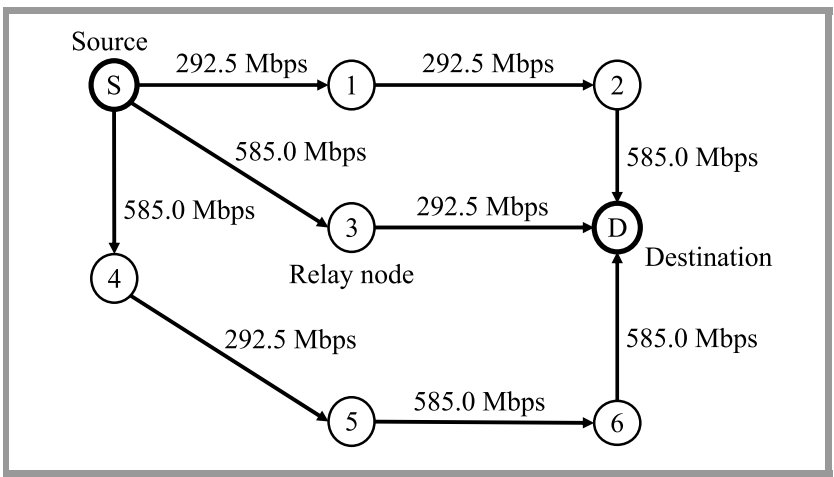

Fig. 6. Simulation topology utilizing 802.11 ac and the available bandwidth when the iteration count is 2000 (default is 292.5 Mbps).

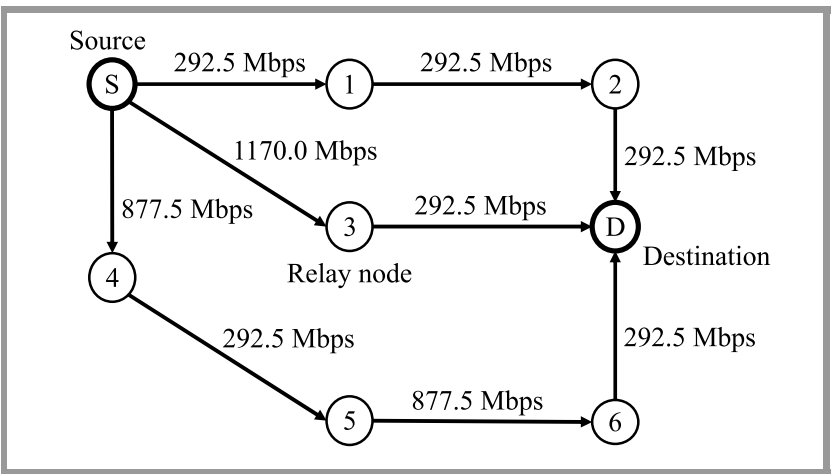

Fig. 7. Simulation topology utilizing 802.11 ac and the available bandwidth when the iteration count is 4000 .

In simulation 4.2, we evaluate the effect of varying available bandwidth using another topology as shown in Figs. 8 and 9. Unlike in the above simulations, in the topologies of the simulation shown in Figs. 8 and 9 relay nodes have

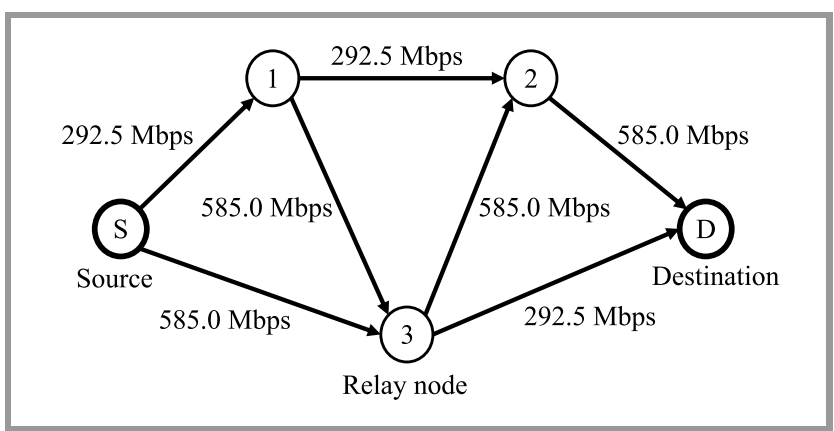

Fig. 8. Simulation topology and the available bandwidth when the iteration count is 2000 (default is $292.5 \mathrm{Mbps}$ ).

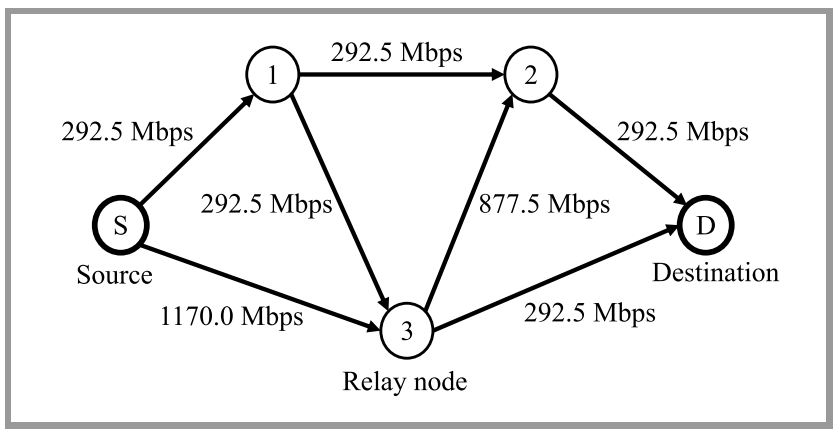

Fig. 9. Simulation topology and the available bandwidth when the iteration count is 4000 .

three or more links. Therefore, we observe the changes in the convergence behavior of the proposed method, since the network topology changes.

\subsection{Simulation Results}

The results of the four simulations described in 4 are shown in Figs. 10 to 12, Fig. 13, Figs. 14 and 15, and Figs. 16 and 17 , respectively. The simulations sufficiently iterate the calculation to observe the effect of convergence of the proposed method. Note that the number of iterations may be shortened by aborting the calculation after a certain number of iterations, when the data transfer volume has been sufficiently converged in a practical situation.

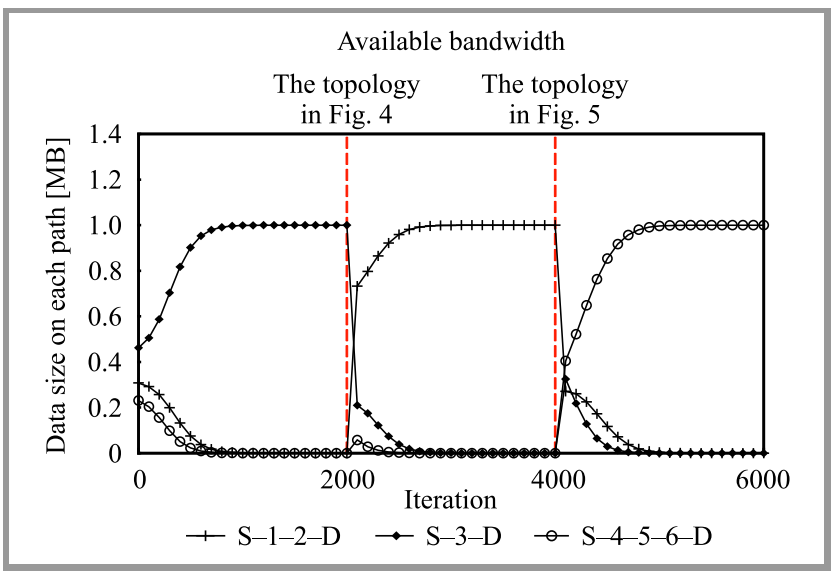

Fig. 10. Simulation 1: the transfer data size when the data transmission volume is fixed at $1 \mathrm{MB}$. 


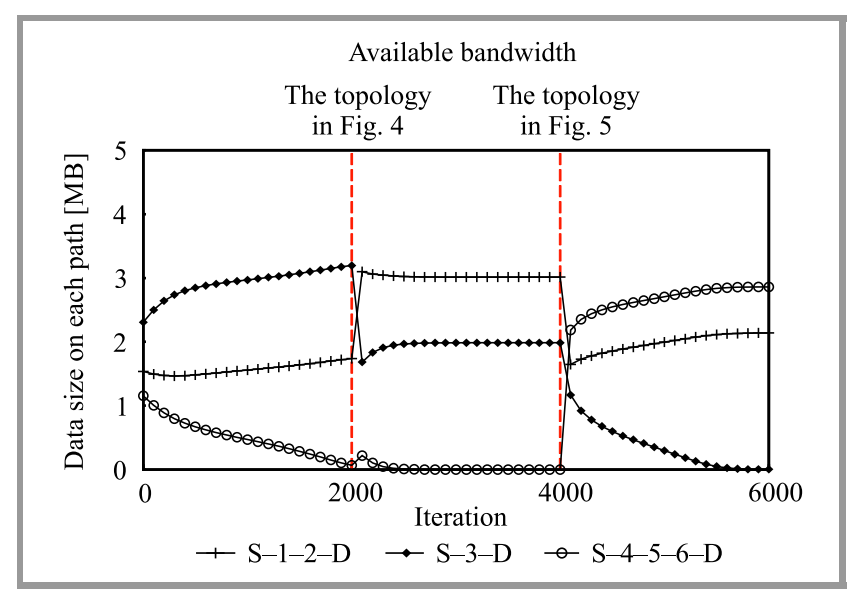

Fig. 11. Simulation 1: the transfer data size when the data transmission volume is fixed at $5 \mathrm{MB}$.

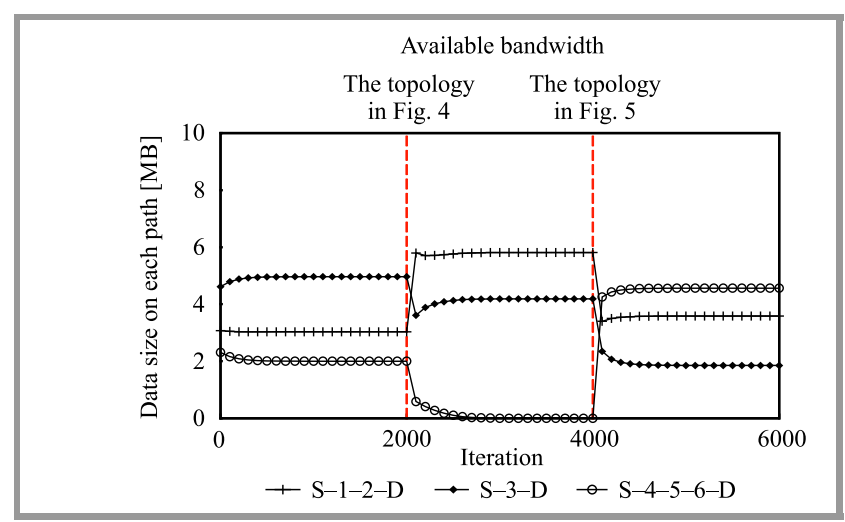

Fig. 12. Simulation 1: the transfer data size when the data transmission volume is fixed at $10 \mathrm{MB}$.

Simulation 1. Figures 10 to 12 show that the data transfer volume of each path increases as the available bandwidth of each link in the path increases. Furthermore, if multiple paths exist, the proposed method balances the required time to transfer data among the paths by calculating their appropriate data transfer volumes based on available bandwidth. In particular, the largest data transfer volume among the paths is allocated to path S-3-D with the minimum hop

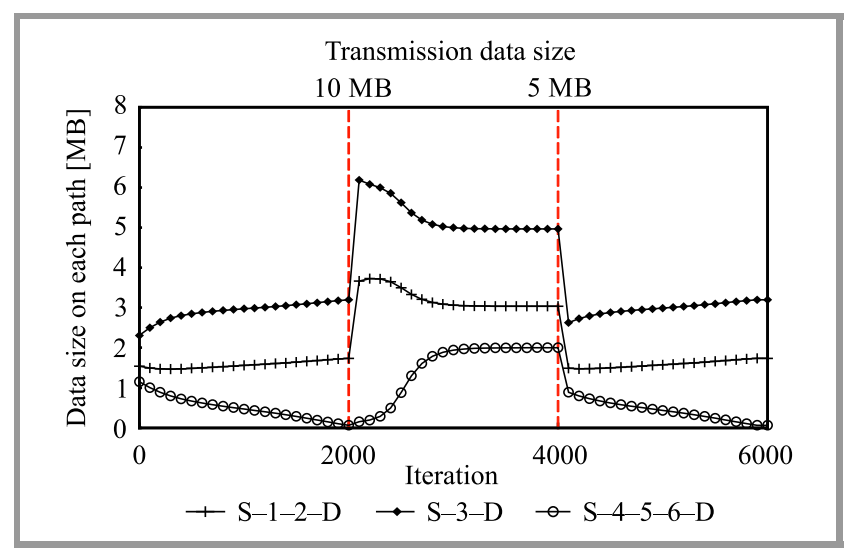

Fig. 13. Simulation 2: data transfer volume when varying data transmission volume. count at the initial state of the simulation. This is because the data transfer volume is preferentially allocated to the path with the minimum transmission delay since the available bandwidth of all links is uniform in the simulation.

Simulation 2. Figure 13 shows that the number of paths increased when the data transfer volume increased. This is because path S-4-5-6-D is constructed as the third path, since the bandwidth occupancy rate of the other two paths is saturated owing to the increase in data size. Additionally, after the data transfer volume is reduced to $5 \mathrm{MB}$, the allocated data size is also decreased and then path S-4-5-6$\mathrm{D}$ disappears. Since the bandwidth occupancy rate of the other two paths gives a bandwidth margin due to a decrease in the data transfer volume.

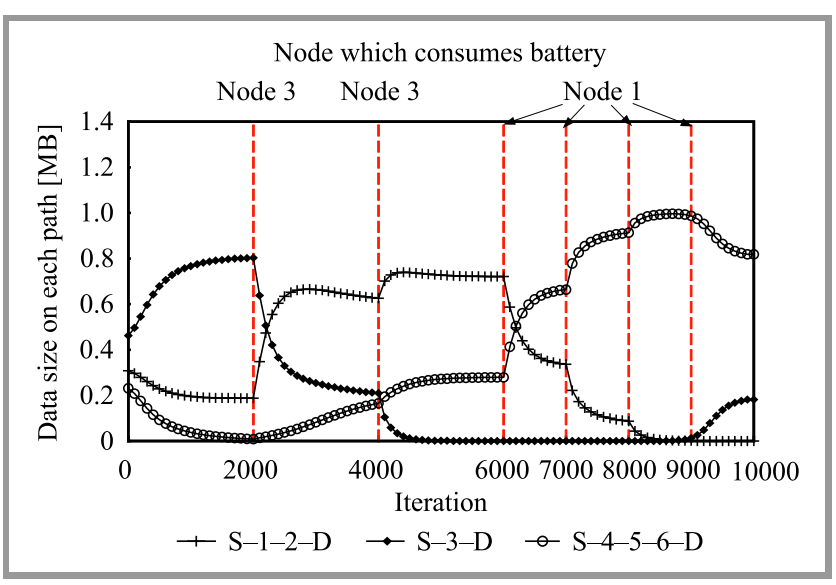

Fig. 14. Simulation 3: data transfer volume when varying residual battery level (data transmission volume: $1 \mathrm{MB}$ ).

Simulation 3. Figure 14 shows that the detour path S-12-D is used since the data transfer volume on path S-3-D via node 3 decreases. In addition, the data transfer volume on path S-4-5-6-D also increases. After, the data transfer data volume on path S-1-2-D decreases because node 1 consumes its battery, when the number of iterations is 6000 . Finally, path S-4-5-6-D, that has a large amount of residual battery of the nodes, is used. Figure 15, shows that paths

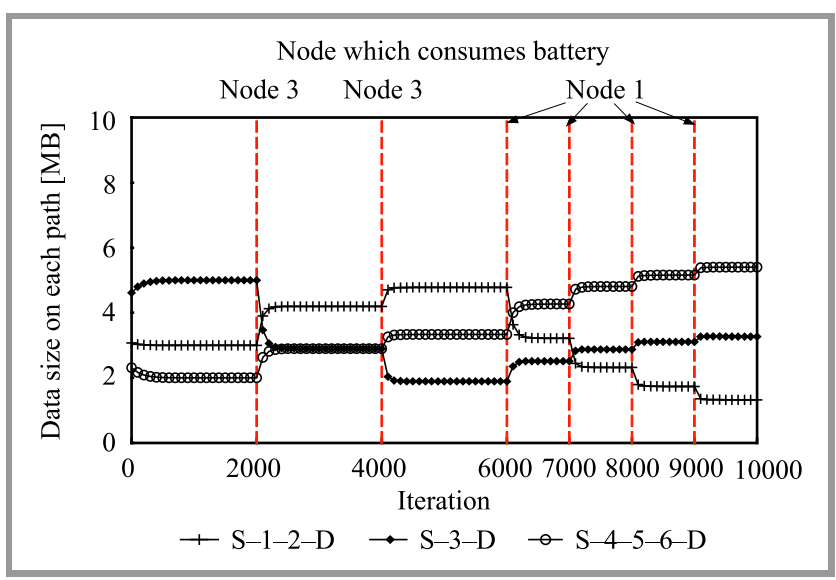

Fig. 15. Simulation 3: the transfer data size when varying residual battery (data transmission volume: $10 \mathrm{MB}$ ). 
S-1-2-D and S-3-D do not disappear, unlike the result in Fig. 14. Since the bandwidth occupancy rate of each path is saturated due to the assigned large data size, the variation of the data transfer volume becomes very small. These results indicate that PS can select routes with a low risk of route disruption due to battery loss.

Simulation 4.1. Figure 16 shows that the path convergence is faster than in simulations 1 to 3 since the variation of the bandwidth occupancy rate reaches its peak at the first calculation step by the sigmoid function due to the large data transfer volume.

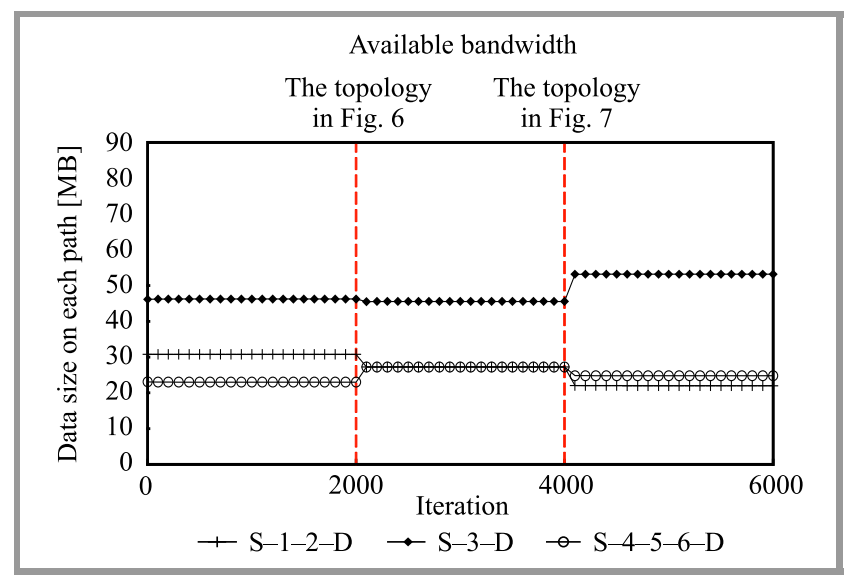

Fig. 16. Simulation 4.1: data transfer volume when the data transmission volume is fixed at $100 \mathrm{MB}$.

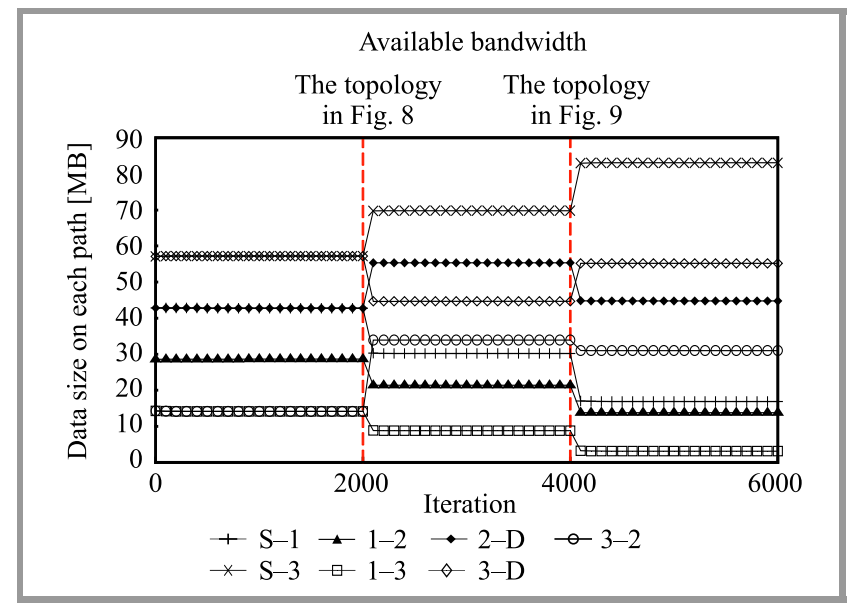

Fig. 17. Simulation 4.2: data transfer volume when the data transmission volume is fixed at $100 \mathrm{MB}$.

Simulation 4.2. Figure 17 shows that the proposed method allocates the data transfer volume based on the available bandwidth of each link while continuing to balance the sent and received data volumes. Additionally, even if the data transfer volume is the same as in simulation 4.1, link 1-3 appears, to which the data transfer volume is scarcely allocated, when the number of iterations is 6000. This is because the number of available paths increased due to the topological change. Hence, these results indicate that the proposed method realizes the allocation of data transfer volume based on the bandwidth available along each path even when a relay node on which the confluence of flows occurs exists in the topology.

\section{Conclusions}

In this paper, we proposed an adaptive ad hoc routing method that can construct multiple paths based on the available bandwidth of each link, data transfer volume and residual battery level of the node by applying PS to the dynamic networks. As a result of our simulations, we confirmed that the proposed method is capable of adaptively constructing single or multiple paths based on the available bandwidth, data transfer volume and residual battery level of nodes, in the dynamic network topology. Our data suggested that PS improves availability of ad hoc network. In the present study, we found that the path convergence time and the number of available paths change according to the number of nodes and links in the topology and the parameters of each link. In the future, the increase in transmission delay due to the increasing of hop counts will be investigated. Moreover, we will define and evaluate the load balancing rate and will propose the parameters needed to optimize the number of paths in various topologies. Additionally, we will conduct a detailed performance evaluation of the proposed method by extension of the existing routing protocol through a network simulator. Furthermore, the proposed method may be extended to be used in a table-driven routing protocol, because the operation of the proposed method requires such network-related information as the maximum bandwidth of the link.

\section{Acknowledgments}

This work was supported by JSPS KAKENHI under Grant Number 17K12680.

\section{References}

[1] M. Shimomura, "The new trends in next generation biomimetics material technology: Learning from biodiversity", Sci. Technol. Trends. Q. Rev., vol. 37, pp. 53-75, 2010 (doi: 11035/2843).

[2] D. Chen, Y. Liu, H. Chen, and D. Zhang, "Bio-inspired drag reduction surface from sharkskin", Biosurf. and Biotribol., vol. 4, no. 2, pp. 39-45, 2018 (doi: 10.1049/bsbt.2018.0006).

[3] S. Das, M. Bhowmick, S. K. Chattopadhyay, and S. Basak, "Application of biomimicry in textiles", Curr. Sci., vol. 109, no. 5, pp. 893-901, 2015 (doi: 10.18520/v109/i5/893-901).

[4] T. Nakagaki, H. Yamada, and A. Tóth, "Path finding by true morphogenesis in an amoeboid organism", Biophys. Chemistry, vol. 92, no. 1, pp. 47-52, 2001 (doi: 10.1016/S0301-4622(01)00179-X).

[5] G.-D. Caro, F. Ducatelle, and L.-M. Gambardella, "AntHocNet: an adaptive nature-inspired algorithm for routing in mobile ad hoc networks", Eur. Trans. on Telecommun., vol. 16, pp. 443-455, 2005, (doi: 10.1002/ett.1062).

[6] R. Leidenfrost and W. Elmenreich, "Firefly clock synchronization in an 802.15.4 wireless network", EURASIP J. on Embed. Syst., vol. 2009, article no. 7, 2009 (doi: 10.1155/2009/186406). 
[7] M. Hato, T. Ueda, K. Kurihara, and Y. Kobatake, "Phototaxis in true slime mold physarum polycephalum", Cell Struct. \& Funct., vol. 1, no. 3, pp. 269-278, 1976 (doi: 10.1247/csf.1.269).

[8] S. Corson and J. Macker, "Mobile ad hoc networking (MANET): routing protocol performance issues and evaluation considerations", RFC 2501, IETF, 1999 (doi: 10.17487/RFC2501).

[9] "IEEE standard 802.11. Wireless LAN medium access control (MAC) and physical layer (PHY) specifications", IEEE Std 802.11, June 1999.

[10] "IEEE Standard for Information technology - Telecommunications and information exchange between systems Local and metropolitan area networks - Specific requirements - Part 11: Wireless LAN Medium Access Control (MAC) and Physical Layer (PHY) Specifications - Amendment 4: Enhancements for very high throughput for operation in bands below $6 \mathrm{GHz}$, IEEE Std 802.11ac-2013, Dec. 2013.

[11] A. Kamerman and L. Monteban, "WaveLAN-II: a high-performance wireless LAN for the unlicensed band", Bell Labs Tech. J., vol. 2, no. 3, pp. 118-133, 2002 (doi: 10.1002/bltj.2069).

[12] R. Karmakar, S. Chattopadhyay, and S. Chakraborty, "Impact of IEEE 802.11n/ac PHY/MAC high throughput enhancements on transport and application protocols - A Survey", IEEE Commun. Surveys \& Tutor., vol. 19, no. 4, pp. 2050-2091 (doi: 10.1109/COMST.2017.2745052).

[13] G. Holland, N. Vaidya, and P. Bahl, "A rate-adaptive MAC protocol for multi-hop wireless networks", in Proc. 7th Ann. Int. Conf. on Mob. Comput. \& Netw. MobiCom'01, Rome, Italy, 2001, pp. 236-251 (doi: 10.1145/381677.381700).

[14] M. Lacage, H. Manshaei, and T. Turletti, "IEEE 802.11 rate adaptation: a practical approach", in Proc. 7th ACM Int. Symp. on Model., Anal., and Simul. of Wirel. and Mob. Syst. MSWiM 2004, Venice, Italy, 2004, pp. 126-134 (doi: 10.1145/1023663.1023687).

[15] X, Wang and C. Li, "A topology-independent broadcasting protocol in ad hoc networks with MIMO links", in Proc. Int. Conf. on Commun. Mob. Comput. CMC 2010, Shenzhen, China, 2010, vol. 3 , pp. 219-223 (doi: 10.1109/CMC.2010.291).

[16] A. Tero, R. Kobayashi, and T. Nakagaki, "A mathematical model for adaptive transport network in path finding by true slime mold", J. of Theor. Biology, vol. 244, no. 4, pp. 553-564 (doi: 10.1016/j.jtbi.2006.07.015).

[17] A. Tero et al., "Rules for biologically inspired adaptive network design”, Science, vol. 327, no. 5963, pp. 439-442, 2010 (doi: 10.1126/science.1177894).

[18] A. Tero, R. Kobayashi, and T. Nakagaki, "Physarum solver: a biologically inspired method of road-network navigation", Physica A: Statis. Mechan. and its Appl., vol. 363, no. 1, pp. 115-119, 2006 (doi: 10.1016/j.physa.2006.01.053).

[19] M. Zhang, W. Wei, R. Zheng, and Q. Wu, "P-bRS: a physarumbased routing scheme for wireless sensor networks", The Scientific World J., vol. 2014, Article ID 531032, 2014 (doi: 10.1155/2014/531032).

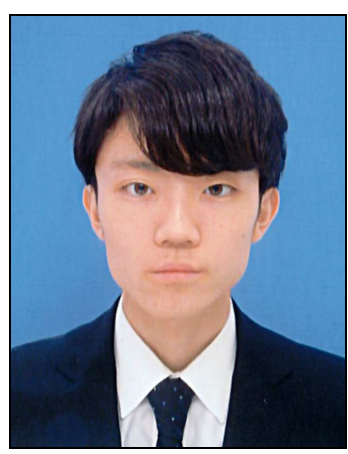

Hiroshi Katada received his B.E. degree in Electronic Information Systems from Shibaura Institute of Technology, Tokyo, Japan, in 2018. He is presently a master's course student at the Graduate School of Engineering and Science, Shibaura Institute of Technology, Saitama, Japan. His research interests include mobile ad hoc networks and engineering neo-biomimetics.

E-mail: mf18024@shibaura-it.ac.jp

Graduate School of Systems Engineering and Science Shibaura Institute of Technology

Saitama, Japan

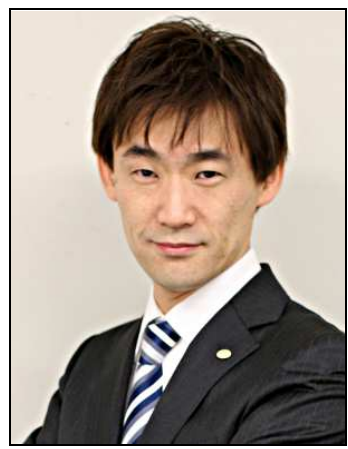

Takumi Miyoshi received his B.E., M.E., and Ph.D. degrees in Electronic Engineering from the University of Tokyo, Tokyo, Japan, in 1994, 1996, and 1999, respectively. He started his career as a research associate at Global Information and the Telecommunication Institute, Waseda University, where he worked from 1999 to 2001.

He is presently a Professor at the Department of Electronic Information Systems, College of Systems Engineering and Science, Shibaura Institute of Technology, Saitama, Japan. He was a visiting scholar in Laboratoire d'Informatique de Paris 6 (LIP6), Sorbonne Université, Paris, France, from 2010 to 2011. His research interests include content delivery networks, overlay networks, as well as mobile ad hoc and sensor networks.

E-mail: miyoshi@shibaura-it.ac.jp

College of Systems Engineering and Science

Shibaura Institute of Technology

Saitama, Japan

Taku Yamazaki - for biography, see this issue, p. 12. 